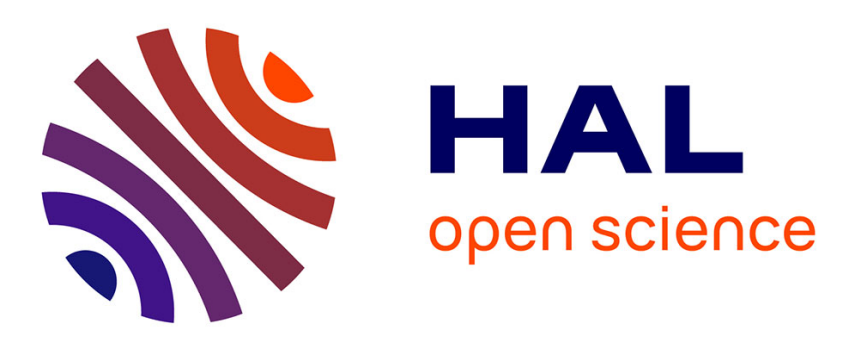

\title{
ÉTUDE DES PROPRIÉTÉS \\ CRISTALLOGRAPHIQUES, MAGNÉTIQUES ET ÉLASTIQUES DE RbCoF3
}

Y. Allain, J. Denis, A. Herpin, J. Lecomte, P. Meriel, J. Nouet, F. Plicque, A. Zarembovitch

\section{To cite this version:}

Y. Allain, J. Denis, A. Herpin, J. Lecomte, P. Meriel, et al.. ÉTUDE DES PROPRIÉTÉS CRISTALLOGRAPHIQUES, MAGNÉTIQUES ET ÉLASTIQUES DE RbCoF3. Journal de Physique Colloques, 1971, 32 (C1), pp.C1-611-C1-613. 10.1051/jphyscol:19711207 • jpa-00214032

HAL Id: jpa-00214032

https://hal.science/jpa-00214032

Submitted on 1 Jan 1971

HAL is a multi-disciplinary open access archive for the deposit and dissemination of scientific research documents, whether they are published or not. The documents may come from teaching and research institutions in France or abroad, or from public or private research centers.
L'archive ouverte pluridisciplinaire HAL, est destinée au dépôt et à la diffusion de documents scientifiques de niveau recherche, publiés ou non, émanant des établissements d'enseignement et de recherche français ou étrangers, des laboratoires publics ou privés. 


\title{
ÉTUDE DES PROPRIETTÉS CRISTALLOGRAPHIQUES, MAGNÉTIQUES ET ÉLASTIQUES DE $\mathrm{RbCoF}_{3}$
}

\author{
par \\ Y. ALLAIN $(*)$, J. DENIS $(*)$, A. HERPIN $(*)(* *)$, J. LECOMTE $(*)$, \\ P. MERIEL $(*)$, J. NOUET $(* * *)$, F. PLICQUE $(* *)$, A. ZAREMBOVITCH $(* *)(* * *)$
}

\begin{abstract}
Résumé. - Une étude aux rayons $\mathrm{X}$ a montré qu'en dessous de $101 \mathrm{\circ K}, \mathrm{RbCoF}_{3}$ devient faiblement quadratique $\left(c / a=0,9971\right.$ à $\left.4,2^{\circ} \mathrm{K}\right)$. Des mesures par diffraction de neutrons ont confirmé qu'au-dessous de $98 \pm 5^{\circ} \mathrm{K}, \mathrm{RbCoF}_{3}$ devenait antiferromagnétique, les moments des atomes de cobalt formant un arrangement de type $\mathrm{G} . \mu_{\mathrm{co}}=3,0 \pm 0,2 \mu_{\mathrm{B}}$ à $4,2^{\circ} \mathrm{K}$. La susceptibilité magnétique suit entre 300 et $600^{\circ} \mathrm{K}$ une loi de Curie-Weiss $\left(\theta_{\mathrm{p}}=180^{\circ} \mathrm{K}, n_{\mathrm{efp}}=5,53\right)$. La température de Néel est au voisinage de $100^{\circ} \mathrm{K}$. Les vitesses de propagation des ultra-sons ont été mesurées en fonction de la température pour différentes directions de propagation.
\end{abstract}

Abstract. - X-rays have shown that below $101{ }^{\circ} \mathrm{K}, \mathrm{RbCoF}_{3}$ becomes slightly tetragonal $\left(c / a=0.9971\right.$ at $4.2{ }^{\circ} \mathrm{K}$ ). Neutron diffraction measurements confirmed that below $98 \pm 5 \mathrm{oK} \mathrm{RbCoF}_{3}$ is antiferromagnetic. The magnetic configuration is of G-type. $\mu_{\mathrm{co}}=3.0 \pm 0.2 \mu_{\mathrm{B}}$ at $4.2 \circ \mathrm{K}$. The magnetic susceptibility obeys a Curie-Weiss law between 300 and $600 \mathrm{~K}\left(\theta_{\mathrm{p}}=180 \mathrm{~K}, n_{\mathrm{efq}}=5.53\right)$. The Néel temperature is in the neighbourhood of $100 \% \mathrm{~K}$. Velocities at which ultrasonic waves propagate have been measured as a function of temperature for different directions.

I. Introduction. - En utilisant des techniques variées, nous avons étudié l'évolution avec la température des propriétés cristallographiques, magnétiques et élastiques de la perovskite fluorée $\mathrm{RbCoF}_{3}$. Les études structurales entreprises antérieurement ont été faites principalement sur les perovskites de potassium $\left(\mathrm{KCoF}_{3}, \mathrm{KNiF}_{3}, \mathrm{KMnF}_{3}, \mathrm{KFeF}_{3}\right)$ : étude cristallographique [1] [2], étude magnétique par mesure de susceptibilité [3] et par diffraction de neutrons [4], et, pour $\mathrm{KMnF}_{3}$, étude des constantes élastiques [5]. A noter également l'étude des propriétés élastiques de $\mathrm{RbMnF}_{3}$ [6] [7]. $\mathrm{RbCoF}_{3}$, comme les perovskites précédentes, devient antiferromagnétique à basse température. La température de Néel serait 96 oK d'après des mesures de RMN [8].

II. Préparation. - La préparation de $\mathrm{RbCoF}_{3}$ a été réalisée dans un flux de chlorure suivant la réaction :

$3 \mathrm{CoF}_{2}+5 \mathrm{RbCl} \rightarrow 2 \mathrm{RbCoF}_{3}+3 \mathrm{RbCl}+\mathrm{CoCl}_{2}$.

On obtient des cristaux qui peuvent être facilement séparés du bain de chlorure. Dans une deuxième étape, la croissance par la méthode Bridgman-Stockbarger, effectuée en tube scellé de platine, permet d'obtenir des échantillons monocristallins de qualité optique [9].

III. Etude cristallographique. - A température ambiante, $\mathrm{RbCoF}_{3}$ est cubique, du type perovskite (groupe d'espace $\operatorname{Pm} 3 \mathrm{~m}, \mathrm{O}_{\mathrm{h}}^{1}$ ). Sa constante réticulaire, déterminée sur poudre est

$$
a_{c}=4.1270 \pm 0,0002 \AA \text {. }
$$

A l'aide d'un diffractomètre à rayons $\mathrm{X}$ équipé d'un cryostat à hélium liquide [10], nous avons mis en évidence une déformation quadratique de la maille

(*) DPH-G/PSRM, C. E. N., Saclay, 91, Gif-sur-Yvette. (**) Faculté des Sciences de Paris.

$(* * *)$ C. S. U., Le Mans. cristalline en dessous de $101^{\circ} \mathrm{K}$. Le rapport $c / a$ atteint 0,9971 à 4,2 oK (Fig. 1).

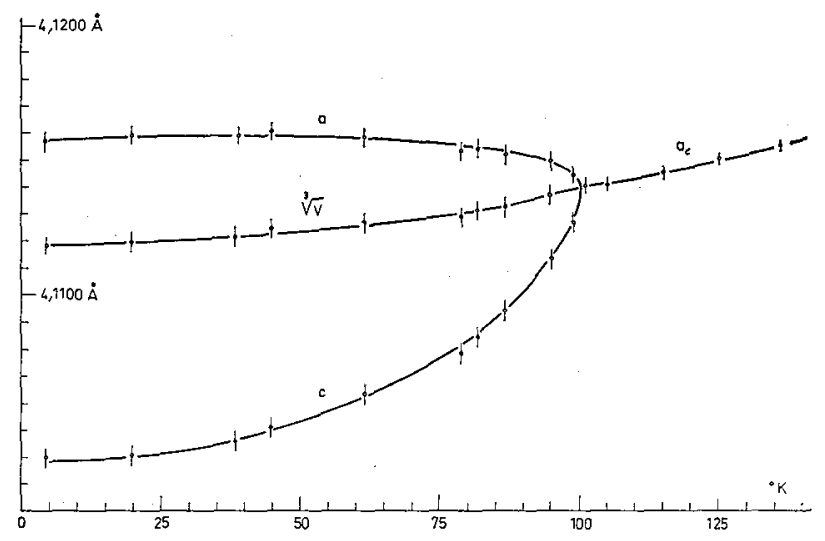

FIG.1. - Variation en fonction de la température des paramètres quadratiques $a$ et $c$, du "paramètre moyen 》 quadratique $\sqrt[3]{v}\left(v\right.$, volume de la maille) et du paramètre cubique $a_{c}$.

IV. Propriétés magnétiques. - $\mathrm{La}$ susceptibilité magnétique de $\mathrm{RbCoF}_{3}$ suit, entre 300 et $600^{\circ} \mathrm{K}$ une loi de Curie-Weiss, avec une température de Curie asymptotique $\theta_{\mathrm{p}}=180^{\circ} \mathrm{K}$ et un nombre efficace de magnétons de Bohr $n_{\text {eff }}=5,53$ (Fig. 2). En dessous de $300^{\circ} \mathrm{K}$, la loi de Curie-Weiss n'est plus vérifiée. La température de Néel se situe entre 100 et 120 \% : la difficulté à déterminer le minimum de la courbe $1 / \chi(T)$ ne permet pas de préciser davantage cette température.

Un spectre de diffraction de neutrons montre qu'à $4,2^{\circ} \mathrm{K}$ le réseau des atomes de cobalt est magnétiquement ordonné suivant une configuration antiferromagnétique de type $\mathrm{G}$ [11], mais ce spectre, réalisé sur poudre, ne permet pas de déterminer l'orientation des moments magnétiques par rapport aux axes du cristal. A partir des raies nucléaires (en prenant $0,70 \times 10^{-12} \mathrm{~cm}$ pour valeur de l'amplitude de diffusion de $\mathrm{Rb}$ [12]), on trouve pour valeur du moment 


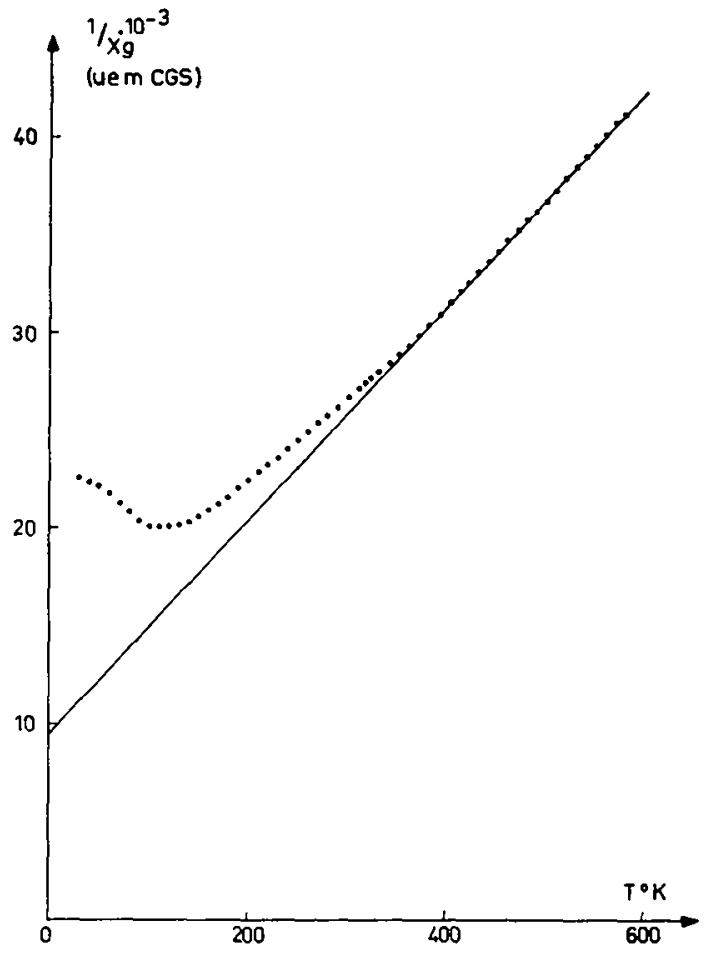

FIG. 2. - Variation avec la température de l'inverse de la susceptibilité magnétique de $\mathrm{RbCoF}_{3}$ (en u.e.m. par gramme).

magnétique du cobalt à $4,20 \mathrm{~K}: 3,0 \pm 0,2 \mu_{\mathrm{B}}$. La figure 3 montre comment varie avec la température l'intensité de la première raie antiferromagnétique. On en déduit par extrapolation $T_{\mathrm{N}}=98^{\circ} \mathrm{K}$ avec une précision qu'on peut estimer à $5^{\circ}$, en bon accord avec la valeur fournie par RMN [9] $\left(96 \pm 1{ }^{\circ} \mathrm{K}\right)$.

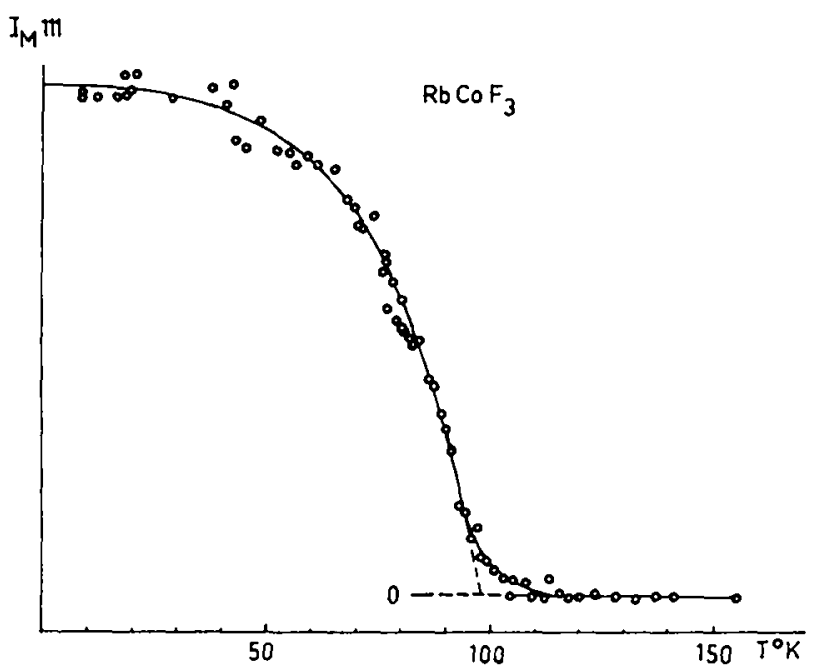

FIc. 3. - Variation de l'intensité de la raie magnétique 111 (dans la maille $2 a, 2 a, 2 c$ ) avec la température.

V. Propriétés élastiques. - Dans la phase cubique, les constantes élastiques du cristal ont été obtenues en mesurant les vitesses de propagation d'ondes longitudinales et transversales se propageant suivant les directions [100], [110], [111] de trois échantillons monocristallins. A température ambiante, deux techni- ques de mesure indépendantes sont mises en œuvre : une technique d'impulsion ultrasonore et une technique de résonance en ondes entretenues. L'évolution des constantes élastiques avec la température est suivie en utilisant la méthode d'impulsion depuis $300^{\circ} \mathrm{K}$ jusqu'à $10^{\circ} \mathrm{K}$. La température de l'échantillon peut être connue à $1^{\circ}$ près et stabilisée à $0,02^{\circ}$ près. A $295^{\circ} \mathrm{K}$ :

$$
\begin{aligned}
c_{11}=(12,96 \pm 0,06) \times 10^{11} \text { dyne } . \mathrm{cm}^{-2} \\
c_{44}=(4,15 \pm 0,02) \times 10^{11} \text { dyne } . \mathrm{cm}^{-2} \\
c_{12}=(5,54 \pm 0,10) \times 10^{11} \text { dyne } . \mathrm{cm}^{-2} \\
\text { avec } \quad \rho_{\text {théor. }}=4,757 \mathrm{~g} . \mathrm{cm}^{-3} .
\end{aligned}
$$

La figure 4 représente la variation avec la température des constantes élastiques dans la phase cubique et, quand les mesures de vitesse sont possibles, les variations de $\rho V^{2}$ dans la phase quadratique polydomaine. Dans ce cas, les directions des axes quaternaires des
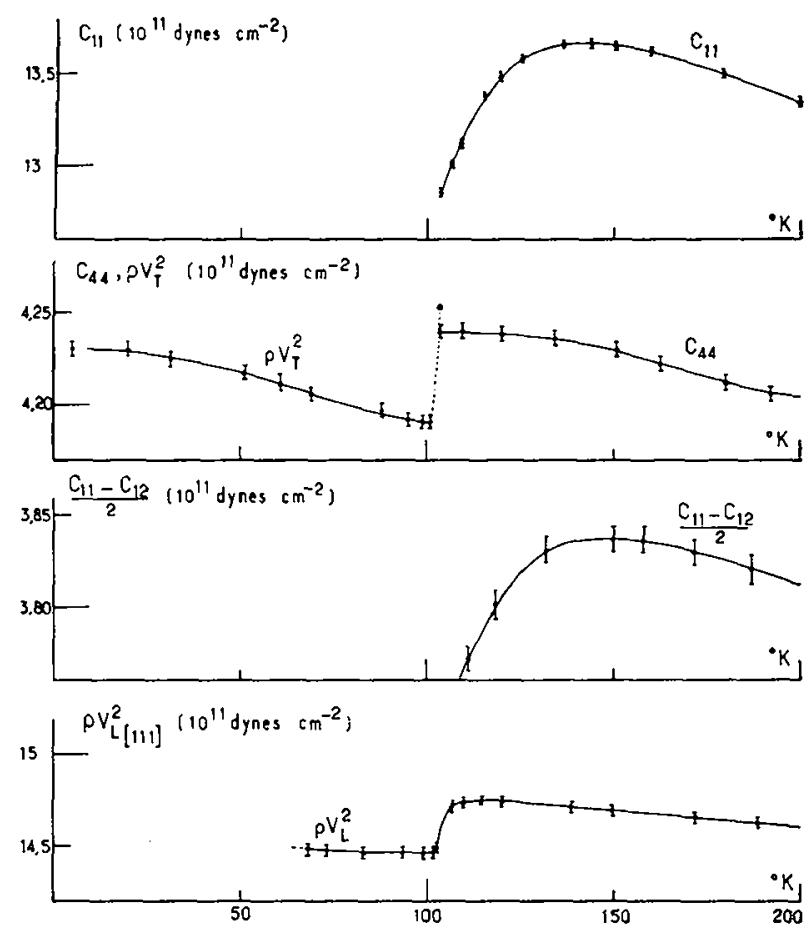

FIg. 4. - Variation des constantes élastiques en fonction de la température dans la phase cubique et dans la phase quadratique polydomaine.

domaines sont distribuées au hasard entre les directions [100], [010], [001]. Il est possible cependant d'obtenir des échos d'impulsions ultrasonores et d'effectuer des mesures de vitesse pour des ondes transversales se propageant suivant la direction [100], et des ondes longitudinales se propageant suivant [111] (un résultat analogue a déjà été signalé pour $\mathrm{SrTiO}_{3}$ [13]). Par ailleurs, les variations relatives de $c_{11}$ et $c_{44}$ sont importantes $(5 \%$ et $1 \%$ respectivement) quand on passe de 120 à $101{ }^{\circ} \mathrm{K}$.

Conclusion. - Aux erreurs d'expérience près, la température de quadratisation coïncide avec celle où l'on observe une variation brutale des constantes élastiques. Un rapprochement avec les résultats obte- 
nus sur $\mathrm{KMnF}_{3}[5]$ (quadratisation sans ordre magnétique) suggère d'attribuer la variation des constantes élastiques au changement de structure. Par contre, il n'est pas certain que l'apparition de l'ordre magnétique coïncide avec le changement de structure cristalline.
Des mesures de chaleur spécifique ont mis en évidence un seul pic de faible amplitude à $101^{\circ} \mathrm{K}$. Il est donc très probable que la température de Néel et la température de quadratisation coïncident à $T=101 \pm 1 \mathrm{oK}$, la transformation étant du second ordre.

\section{References}

[1] OKazaki (A.), Suemene (Y.), Fuchikami (T.), $J$. Phys. Soc. Japan, 1959, 14, 1823.

[2] Beckman (O.), KNox (K.), Phys. Rev., 1961, 121, 376.

[3] Hirakawa (K.), Hashimoto (T.), J. Phys. Soc. Japan, $1960,15,2063$.

[4] Scatturin (V.), Corliss (L.), Hastings (J.), Acta Cryst., 1961, 14, 19.

[5] Aleksandrov (K. S.), Reshchikova (L. M.), BeZNOSIKOV (B. V.), Phys. Status Solidi, 1966, 18, $\mathrm{K} 17$.

[6] Melcher (R. L.), Bolef (D. I.), Stevenson (R. W. H.), Solid State Commun., 1967, 5, 735.

[7] Melcher (R. L.), Bolef (D. I.), Phys. Rev., 1969, 178, 864 .
[8] Petrov (M. P.), Nedlin (G. M.), J. Appl. Phys., $1968,39,1012$.

[9] Nouet (J.), JACoboni (C.), Ferey (G.), Gerard (J. Y.), DE PAPE (R.), J. Cryst. Growth (sous presse)

[10] Nouet (J.), KLeINBERGER (R.), De KOUCHKOVSKY (R.), Acad. Sci. Paris, 1969, 269 B, 986.

[11] Wollan (E. O.), Koehler (W. C.), Phys. Rev., 1955, $100,544$.

[12] Copley (J. R. D.), Acta Cryst., 1970, A26, 376. Wang (F. F. Y.), Cox (D. E.), Acta Cryst., 1970 A 26, 377.

Meriel (P.), Acad. Sci. Paris, 1970, B270, 560.

[13] Bell (R. O.), Rupprecht (G.), Phys. Rev., 1963, 129, 90 . 